\title{
Sensor Fusion for Improved Control of Piezoelectric Tube Scanners
}

\author{
Andrew J. Fleming, Member, IEEE, Adrian G. Wills, and S. O. Reza Moheimani, Senior Member, IEEE
}

\begin{abstract}
In nanopositioning applications, capacitive or inductive sensors are used to measure displacement and provide feedback to eliminate actuator nonlinearity, dynamics, cross-coupling between axes, and thermal drift. Due to their noise density, typically $20 \mathrm{pm} / \sqrt{\mathrm{Hz}}$ for $100-\mu \mathrm{m}$ range transducers, feedback loops are restricted to a few tens of Hertz if nanometer precision is required. In this study, a capacitive displacement sensor is used with a piezoelectric strain voltage measurement to reduce sensor noise at frequencies above $1 \mathrm{~Hz}$. The piezoelectric strain voltage is derived from an open-circuit electrode on a four-quadrant piezoelectric tube actuator and requires no additional hardware. The noise density of the piezoelectric strain voltage is measured to be three orders of magnitude lower than the capacitive sensor. This allows a large increase in closed-loop bandwidth with no penalty on sensor-induced noise. The advantageous properties of the capacitive sensor and piezoelectric strain voltage are discussed and utilized to design a Kalman filter that combines the two signals in a statistically optimal way. A receding horizon control strategy is then introduced as a technique for controlling the tube scanner. A wide-bandwidth controller is implemented that provides reference tracking and damping of the actuator resonance, with root-mean-square displacement noise below $0.4 \mathrm{~nm}$.
\end{abstract}

Index Terms-Displacement estimation, Kalman filter, nanopositioner, piezoelectric strain sensor, piezoelectric tube scanner, receding horizon control, sensor fusion.

\section{INTRODUCTION}

A PIEZOELECTRIC tube scanner is a thin cylinder of radially poled piezoelectric material with four external electrodes and a grounded internal electrode. A tube scanner develops a lateral tip deflection in response to an applied voltage. When a voltage is applied to one of the external electrodes, the actuator wall expands and, due to Poisson coupling, causes a vertical contraction and large lateral deflection of the tube tip. A piezoelectric tube with an aluminum base and end cap is pictured in Fig. 1. A typical application diagram is shown in Fig. 2, where a displacement sensor and feedback loop are used to reduce nonlinearity and regulate dynamics.

In the following, the difficulties associated with piezoelectric tube scanners are discussed. The strengths and limitations of present control techniques are then surveyed. This leads us

Manuscript received October 18, 2007. Manuscript received in final form January 31,2008 . First published June 10, 2008; current version published October 22, 2008. Recommended by Associate Editor S. Devasia. This work was supported in part by the Australian Research Council under Discovery Project DP0666620, by the University of Newcastle, and by the ARC Center for Complex Dynamic Systems and Control.

The authors are with the School of Electrical Engineering and Computer Science, University of Newcastle, Callaghan, NSW 2308, Australia (e-mail: andrew.fleming@ newcastle.edu.au).

Color versions of one or more of the figures in this paper are available online at http://ieeexplore.ieee.org.

Digital Object Identifier 10.1109/TCST.2008.921798

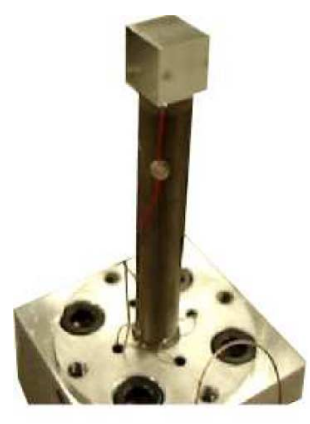

(a)

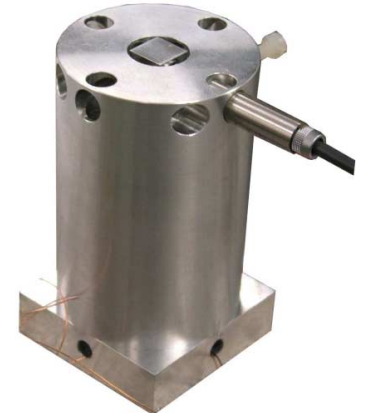

(b)
Fig. 1. (a) Piezoelectric tube mounted inside (b) an aluminum shield. The $x$-axis capacitive sensor is shown secured at right angles to a cube mounted onto the tube tip.

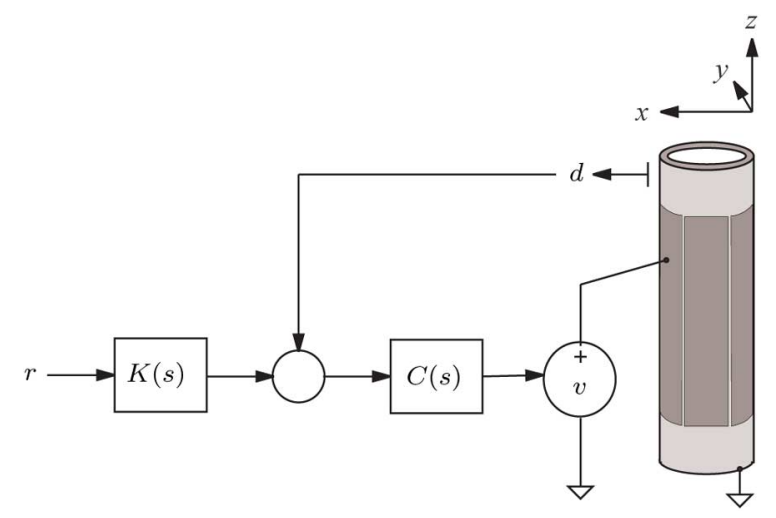

Fig. 2. Voltage driven tube scanner with reference input $r$, feedforward filter $K(s)$, displacement measurement $d$, and feedback controller $C(s)$.

to propose a feedback control approach with the use of a new sensor arrangement that significantly reduces sensor noise and increases dynamic range. A standard capacitive position sensor is combined with a strain measurement obtained from an opencircuited tube electrode to provide a high bandwidth and lownoise position estimate.

\section{A. Difficulties With Tube Scanners}

Piezoelectric tubes are used extensively in applications requiring precision positioning such as scanning probe microscopy [1]-[4], nanofabrication systems [5], [6], and nanomanipulation devices [7], [8]. In these applications, piezoelectric tubes are designed with large length-to-diameter ratios, as this provides a large lateral deflection range but imposes low mechanical resonance frequencies. In nanometer precision raster scanning applications, such as scanning probe microscopy, the maximum triangular scan rate is limited to around $1 \%-10 \%$ of the first resonance frequency. To illustrate 
the problem, consider a typical piezoelectric tube with a maximum lateral deflection of $100 \mu \mathrm{m}$ and a resonance frequency of $700 \mathrm{~Hz}$. For a scanning probe microscope, this equates to more than one minute of image acquisition time (at $640 \times 480$ resolution) and severe throughput limitations in nanomanipulation and fabrication processes.

Nonlinearity is another ongoing difficulty associated with piezoelectric tube scanners (and piezoelectric actuators in general). When employed in an actuating role, piezoelectric transducers display significant creep and hysteresis in response to an applied voltage [9]. Due to nonlinearity, ideal scanning signals can result in severely distorted tip displacements and, hence, poor image quality and poor repeatability in nanofabrication processes.

\section{B. Techniques for Tracking-Error Reduction}

Techniques aimed at addressing both mechanical dynamics and hysteresis can be grouped generally into two broad categories, feedforward and feedback. Feedforward techniques do not include a sensor but require accurate knowledge of the nonlinearity and/or dynamics. Feedback systems, although more robust to modeling error, are limited by the noise performance and bandwidth of the sensor. In many cases, it is also difficult and/or prohibitively expensive to integrate displacement sensors into the scanning apparatus.

Feedforward and signal compensation approaches have been extensively studied as their implementation requires no additional hardware or sensors. It should be considered, however, that additional hardware such as displacement sensors and DSP processors are required to identify the behavior of each tube prior to implementation. A technique for designing optimal linear feedforward compensators was presented in [10] and then later extended to incorporate a PD feedback controller in [11]. In these works, the authors identify the main limitation to performance as modeling error. Another feedforward technique, known as iterative or learning control, is aimed at reducing unmodeled hysteresis. In this approach the need for a model is essentially annulled with the use of a sensor and online iteration to ascertain the optimal input compensation [12], [13]. The foremost problems with iterative techniques are the time taken to iterate the compensator and inability to control disturbance and cross coupling. Other feedforward approaches have included optimal $\mathcal{H}_{\infty}$ compensation [14], compensation for creep, Preisach hysteresis, and resonance [15], improved iterative Preisach inversion [16], and various optimal linear feedforward compensation techniques [17], [18].

Feedback control of piezoelectric tube scanners, which was first proposed by Tamer and Dahleh in 1994 [19], can provide excellent low-frequency tracking performance but is heavily dependent on the sensor noise performance and bandwidth. Such techniques are most applicable to scan ranges in the hundreds of nanometers or greater where sensor noise is more tolerable. Good tracking of a $5-\mathrm{Hz}$ triangle wave while maintaining robustness to nonlinearity was reported in [20]. With the integration of displacement sensors into the next generation of commercial microscopes, feedback systems are becoming more popular.
Feedback control of piezoelectric tube scanners and nanopositioners is generally accomplished with the aid of a capacitive, inductive, or optical displacement sensor. With the exception of interferometers which are prohibitively expensive for commercial applications, and if the target can be adequately grounded, capacitive sensors offer the greatest resolution and signal-to-noise ratio (SNR). Again, with the exception of interferometers, all of the techniques mentioned are severely limited in bandwidth if high resolutions or dynamic ranges are required. As an example, consider a high-performance capacitive sensor with a range of $\pm 100 \mu \mathrm{m}$ and a root-mean-square (rms) noise of $10 \mathrm{pm} / \sqrt{\mathrm{Hz}}$. To achieve an rms noise of $0.1 \mathrm{~nm}$, the bandwidth must be limited to $100 \mathrm{~Hz}$ and to $1 \mathrm{~Hz}$ for atomic resolution on graphite. Consequently, feedback control of scanning tunneling microscopes is not feasible.

\section{Contribution of This Paper}

In this study, we propose a new technique for measuring the displacement of piezoelectric tube scanners. The displacement measurement is obtained from the open-circuit voltage measured on one of the actuator's quadrant electrodes, which is proportional to displacement. This new measurement exhibits wide bandwidth and exceptionally low noise, but is temperature dependant and lacks accuracy at dc.

In this study, we combine a capacitive sensor and piezoelectric strain voltage measurement to yield an extremely high-accuracy displacement estimate. This method possesses the environmental stability of capacitive sensors and the low noise and wide bandwidth of piezoelectric transducers. An rms measurement noise of approximately $0.4 \mathrm{~nm}$, with $100-\mu \mathrm{m}$ range and $20-\mathrm{kHz}$ bandwidth, is demonstrated experimentally. With the aid of a low-variance displacement estimate, it is possible to construct a wide-bandwidth feedback control system with low noise. In this study, receding horizon control is selected for its ease of implementation and suitable objective function, which is to minimize tracking error over a finite horizon.

This paper proceeds with a description of the scanner apparatus in Section II and a discussion of signal properties and displacement estimation in Section III. Receding horizon control is introduced in Section IV, followed by experimental results in Section V.

\section{SCANNING APPARATUS}

The length, diameter, and wall thickness of a piezoelectric tube scanner define the available scan range and mechanical bandwidth. Longer, narrower tubes of around $50-80 \mathrm{~mm}$ are used for achieving large deflections of around $100 \mu \mathrm{m}$, while shorter tubes of around $15 \mathrm{~mm}$ are used for small deflections of $1 \mu \mathrm{m}$ or less. Variations include: a circumferential electrode for independent vertical extension or diameter contraction, and/or sectored internal electrodes. Small deflection expressions for the lateral tip translation can be found in [21]. Measured in the same axis $(x$ or $y)$ as the applied voltage, the tip translation $d$ is approximately

$$
d_{i}=\frac{\sqrt{2} d_{31} L^{2}}{\pi D h} v_{i}, \quad i=x, y
$$




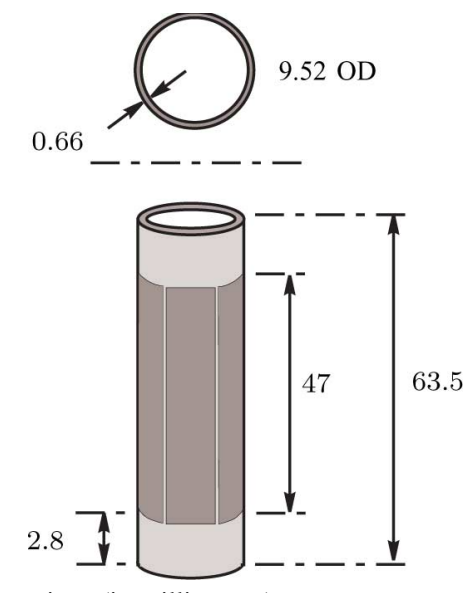

Fig. 3. Tube dimensions (in millimeters).

where $d_{i}$ is the ( $x$-or $y$-axis) deflection, $d_{31}$ is the piezoelectric strain constant, $L$ is the length of the tube, $D$ is the outside diameter, $h$ is the tube thickness, and $v_{i}$ is the ( $x$ - or $y$-axis) electrode voltage. Tip deflection can be doubled by applying an equal and opposite voltage to electrodes in the same axis.

Vertical elongation of the tube due to a voltage applied equally to all four quadrants is given approximately by

$$
\Delta L=\frac{d_{31} L}{h} v
$$

where $\Delta L$ is the change in length [21].

As pictured in Fig. 1, the apparatus used in this study comprises a piezoelectric tube housed in a removable aluminium shield. A polished, hollow aluminium cube $8 \mathrm{~mm}$ square ( $1.5 \mathrm{~g}$ in mass) is glued to the tube tip to allow displacement measurements with an ADE Tech 4810 Gaging Module and 2804 capacitive sensor. The sensitivity of the capacitive sensor is $100 \mathrm{mV} / \mu \mathrm{m}$ over a range of $\pm 100 \mu \mathrm{m}$ and bandwidth of $10 \mathrm{kHz}$. During assembly, the shield serves as a jig to ensure that the tube is both vertical and aligned in the same axis as the cube face and capacitive sensor head. Nylon grub screws secure the sensor heads after assembly.

The tube was manufactured by Boston PiezoOptics from high density PZT-5H piezoelectric ceramic. Relevant physical dimensions can be found in Fig. 3. Four equally spaced quadrant electrodes are deposited around the tube circumference. The electrodes are driven with an in-house $\pm 200 \mathrm{~V}$ charge amplifier [22]. Charge amplifiers have been shown to reduce hysteresis in piezoelectric tube scanners by $89 \%$; see [23] and [22] for details.

The tip displacement frequency response, measured using an HP 35670A spectrum analyzer, is plotted in Fig. 4. The free response has a first resonance at $850 \mathrm{~Hz}$ and a static sensitivity of $171 \mathrm{~nm}$ per volt. To evaluate performance robustness in Sections III-V, a worst case mass of $1.5 \mathrm{~g}$ is affixed to the top cube surface. As shown in Fig. 4, the additional mass reduces the resonance frequency by $110 \mathrm{~Hz}$ or $13 \%$ and increases the damping ratio.

\section{SENSOR DESIGN}

\section{A. Signal Characteristics}

The available displacement signals, capacitive, piezoelectric, and simulated, are illustrated in Fig. 5. The characteristics of

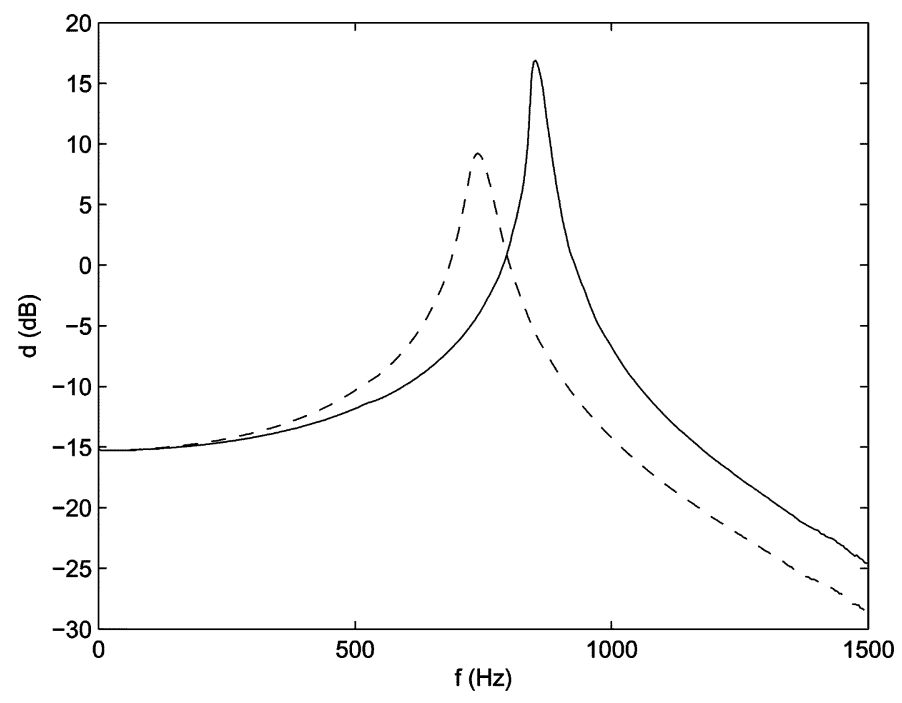

Fig. 4. Scanner frequency response measured from the applied voltage to the tip displacement $d$ (in micrometers per volts); unloaded ( $\mathrm{g}$ compliant mass (--).

each signal are discussed in the following. Capacitive displacement sensing is then compared with piezoelectric strain voltage measurement in Section III-A1.

1) Simulated Displacement $y_{m}(s)=r(s) g(s)$ : The simulated displacement is the result of filtering the measured input $r$ with a dynamic model of the scanner dynamics $G$. Controlling the simulated output $y_{m}$ is equivalent to performing modelbased feedforward control. The output $y_{m}$ is subject to large uncertainties due to model mismatch, temperature variation, and load changes. The sensitivity of $G(s)$ should be periodically calibrated using the capacitive sensor.

2) Capacitive Sensor $y_{c}$ : A capacitive sensor applies a highfrequency potential between two plates, and the resulting current is related to capacitance and displacement. The displacement sensitivity is highly stable and largely invariant to temperature and environmental conditions; it is the most reliable measurement.

As shown in Fig. 6, the displacement signal is the filtered sum of the true displacement $d$ and additive white noise $v_{n c}$. As discussed in the introduction, a low-pass filter $G_{c}$ provides an arbitrary resolution at the expense of bandwidth.

The capacitive sensor provides an accurate method for calibration of the simulated model and strain measurement. Capacitive sensors are also excellent for recording system frequency responses, for example, the transfer function from an applied voltage to displacement. Averaging or swept-sine analysis can be employed to reduce noise to acceptable levels.

3) Piezoelectric Strain Voltage $y_{p}$ : As shown in Fig. 5, the piezoelectric strain voltage $v_{p}$ is proportional to the strain $\varepsilon$ and tip displacement $d$. The constant $k_{\varepsilon}$, relating the piezoelectric strain voltage to tip deflection, is a function of the scanner geometry, material properties, and piezoelectric strain constant.

Due to the high source impedance, especially at low frequencies, care must be taken to avoid contamination by interference and loss due to parasitic capacitance and leakage. An acceptable solution is triaxial cable with the outer sheath grounded at the instrument case and connected to one terminal of the 


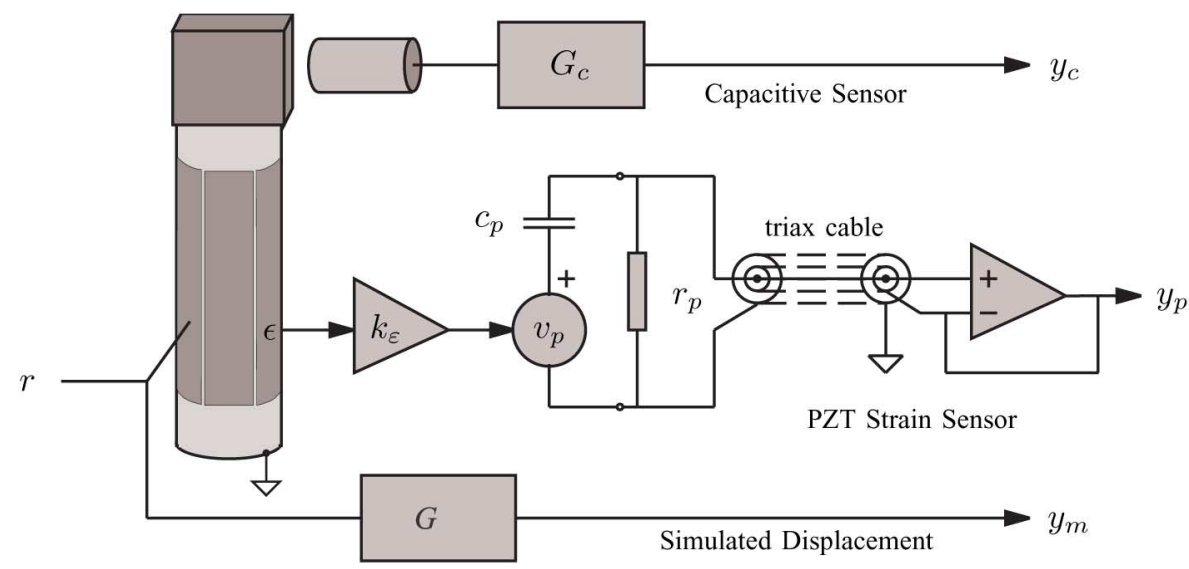

Fig. 5. Displacement measurements from the capacitive sensor $y_{c}$, the piezoelectric strain voltage $y_{p}$, and the simulated displacement $y_{m} . k_{\varepsilon}$ represents the constant relating mechanical strain $\varepsilon$ to the induced piezoelectric voltage $v_{p}$.

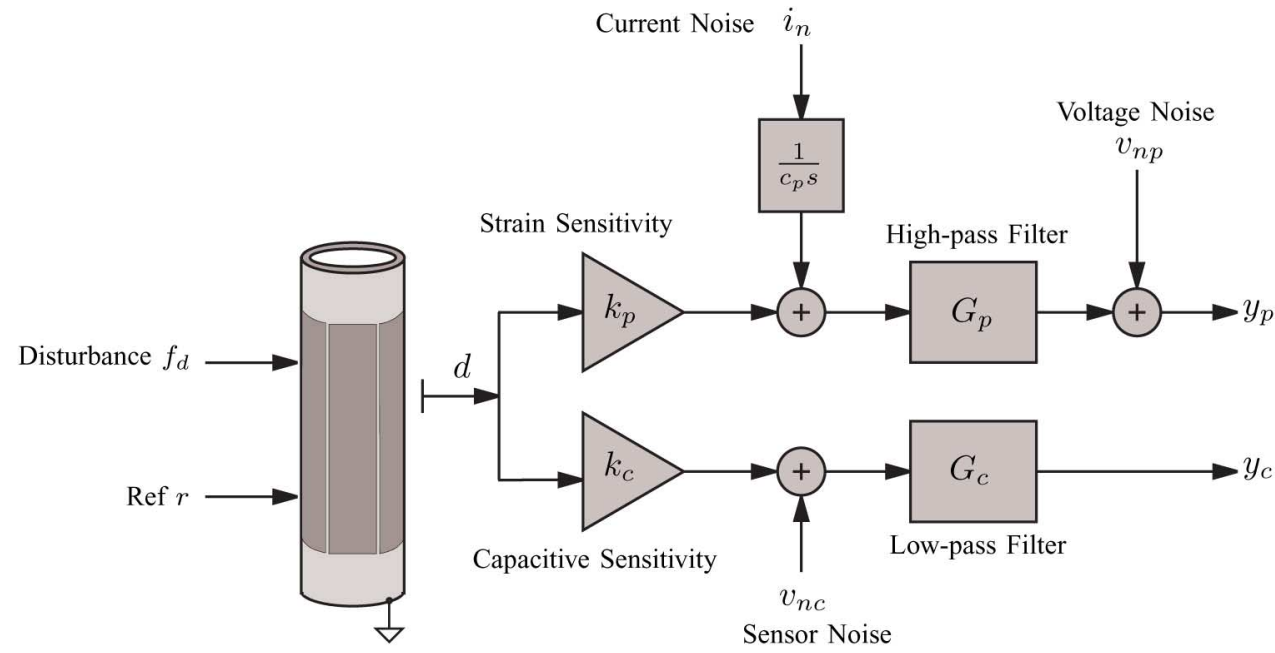

Fig. 6. Signal path and additive noise from the applied voltage to the measured strain voltage and capacitive sensor output. $k_{p}$ and $k_{c}$ represent the sensitivity of the two sensors from displacement to developed voltage.

transducer. The inner conductor is connected to the transducers' high-impedance terminal with an intermediate shield driven by a buffer stage to eliminate parasitic capacitance and leakage. The buffer should be a high-transconductance FET or MOSFET common drain amplifier or FET input op amp.

The transducer capacitance $c_{p}$ together with the lumped dielectric leakage and external resistance $r_{p}$ creates the first-order high-pass filter

$$
G_{p}(s)=\frac{s}{s+\frac{1}{r_{p} c_{p}}} .
$$

The dominant noise processes $v_{n p}$ and $i_{n}$ are the input voltage noise and current noise of the buffer stage, respectively. Due to the high source impedance at frequencies below $1 \mathrm{kHz}$, buffer current noise is of the greatest concern. Although the true current noise filter is a leaky integrator $1 /\left(c_{p} s+1 / r_{p}\right)$ with breakpoint $\omega=1 / r_{p} c_{p}$, it is approximated as a pure integrator $1 / c_{p} s$ as shown in Fig. 6. The justification for this simplification is in the nature of the current noise density. Both the voltage and current noise density increase at lower frequencies with a first- order break-point of around 1-100 Hz. The low frequency increase in current noise density approximately cancels the integrator leakage yielding a white current noise density and pure integrator. Voltage noise is insignificant by comparison at low frequencies.

In addition to random noise, interference from drive signals applied to other axes is a significant source of error. There are two mechanisms that provide coupling between adjacent axes: capacitive electrical coupling and mechanical coupling. Voltages of up to $5 \%$ of the applied drive signal can be induced on adjacent open-circuit electrodes. A technique for reducing this error mechanism is discussed in Section III-E.

The final source of error is nonlinearity between the displacement, strain, and measured voltage. The total harmonic distortion (THD) of the strain voltage was measured at approximately $3 \%$ at tip deflections of $10 \mu \mathrm{m} .^{1}$

${ }^{1}$ Although these results utilize a voltage-calibrated strain sensor, a charge-calibrated circuit where the electrode terminals are effectively short-circuited resulted in a THD of less than $0.5 \%$ at frequencies below $100 \mathrm{~Hz}$. Hydrophones are typically calibrated in the voltage mode whereas accelerometers are calibrated in the charge mode. 
TABLE I

Capacitive Versus Piezoelectric Displacement Sensing

\begin{tabular}{|l|l|l|l|}
\hline & $y_{c}$ & $y_{p}$ & Scaled $y_{p}$ \\
\hline \hline Electrical Interference & No & Yes & Yes \\
\hline Electrostatic Cross-coupling & No & Yes & Yes \\
\hline Mechanical Cross-coupling & No & Yes & Yes \\
\hline Bandwidth & DC-Tens kHz & $\mathrm{Hz}-\mathrm{MHz}$ & $\mathrm{Hz}-\mathrm{MHz}$ \\
\hline Scan Range & $\pm 100 \mu \mathrm{m}$ & Unlimited & Unlimited \\
\hline Sensitivity & $100 \mathrm{mV} / \mu \mathrm{m}$ & $6.2 \mathrm{~V} / \mu \mathrm{m}$ & $100 \mathrm{mV} / \mu \mathrm{m}$ \\
\hline Voltage Noise & $1.75 \mu \mathrm{V} / \sqrt{\mathrm{Hz}}$ & $0.10 \mu \mathrm{V} / \sqrt{\mathrm{Hz}}$ & $1.6 \mathrm{nV} / \sqrt{\mathrm{Hz}}$ \\
\hline Displacement Noise & $17.5 \mathrm{pm} / \sqrt{\mathrm{Hz}}$ & $16 \mathrm{fm} / \sqrt{\mathrm{Hz}}$ & $16 \mathrm{fm} / \sqrt{\mathrm{Hz}}$ \\
\hline
\end{tabular}

\section{B. Capacitive Versus Piezoelectric Displacement Sensing}

The nominal sensitivity of the piezoelectric sensor was measured by applying a $10-\mathrm{V} 30-\mathrm{Hz}$ sinusoid to one of the tube electrodes and recording the induced voltage and corresponding displacement. A low current-noise buffer with an input impedance of $66 \mathrm{M} \Omega$ was used to acquire the strain voltage. In this study, we quantify measurement noise via noise density, measured in units $/ \sqrt{\mathrm{Hz}}$, where units could be volts, nanometers, or something else. The rms noise observed within a given bandwidth is related to noise density by

$$
\text { RMS Noise }=\sqrt{\text { Bandwidth }} \times \text { Noise Density. }
$$

This equation also describes the link between bandwidth and rms noise. It states that a tenfold reduction in rms noise can be achieved at the expense of a hundredfold reduction in signal bandwidth. The noise densities of the capacitive sensor and piezoelectric strain voltage were measured using an HP 35670A spectrum analyzer and homemade low-noise amplifier with a gain of 10000 and high-pass cutoff of $50 \mathrm{MHz}$. A summary of the sensitivities and noise densities can be found in Table I.

The piezoelectric strain voltage exhibits a measurement noise three orders of magnitude below the capacitive sensor. This allows tens of kilohertz bandwidth with only a few picometers of random noise. To the best of the authors' knowledge, this is the lowest noise displacement measurement available for piezoelectric tube scanners by a considerable margin. Unfortunately, even though the random noise is extremely low, the high impedance of the strain voltage measurement adds additional difficulties: it is prone to electrical interference and electrostatic cross coupling between adjacent electrodes. In addition, the strain voltage is highly cross-coupled, that is, it is sensitive to deflections in the adjacent lateral axis. These limitations were discussed more thoroughly in Section III-A3.

\section{Linear Sensor Fusion}

A frequency-weighted sum, depicted in Fig. 7, is the simplest technique for obtaining an improved displacement estimate $\widehat{d}$. After scaling each signal into micrometers, the filters $F_{c}$, $F_{p}$, and $F_{m}$ invert sensor dynamics and contain the frequency weighting filters $W_{c}, W_{p}$, and $W_{m}$. The sum of weighting filters must equal 1 so that $\widehat{d}=d$ when the noise is 0 , i.e.,

$$
W_{c}(j \omega)+W_{p}(j \omega)+W_{m}(j \omega)=1 \forall \omega .
$$

A simple method for reducing the variance in $\widehat{d}$ is to find the frequency $w_{\alpha}$ where the noise density of $y_{c}$ is equal to that of

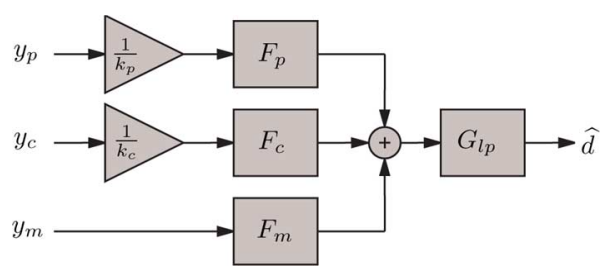

Fig. 7. Sensor fusion by summing the filtered displacement signals. The filters $F_{c}, F_{m}$, and $F_{p}$ are the concatenated weighting filters and sensor equalizers, and $G_{l p}$ is a global low-pass filter used to manipulate the tradeoff between bandwidth and estimate variance.

the strain voltage $y_{p}$; a first-order high- and low-pass weighting is then imposed on the strain voltage and capacitive signal accordingly. The resulting filters $F_{c}$ and $F_{p}$ are

$$
\begin{aligned}
& F_{c}=G_{c}^{-1} \frac{\omega_{\alpha}}{s+\omega_{\alpha}} \\
& F_{p}=\frac{s+\frac{1}{r_{p} c_{p}}}{s} \frac{s}{s+\omega_{\alpha}}
\end{aligned}
$$

where the first and second term in each expression are the sensor equalizer and weighting filter, respectively. In this case, $F_{m}=0$ and the weighting filters are first order.

To ensure causality of the filter network, the relative degree of the global low-pass filter $G_{l p}$ must be equal to or greater than the relative degree of $G_{c}$ minus the relative degree of the weighting filters, in this case 1 . Due to the integrated current noise associated with the piezoelectric strain voltage, higher order weighting filters are beneficial, but more difficult to implement.

In most cases where force disturbances on the scanner are significant, the simulated output $y_{m}$ is of little interest. However, it can become particularly useful in practical environments where correlated interference is present. Mains frequency interference in the piezoelectric strain voltage can be problematic. As interference is present only in the measured variable, not the physical process, a narrowband filter weighting on $y_{m}$ can significantly reduce the overall measurement noise. A unity-peak second-order resonant filter is suitable for such a purpose with transfer function

$$
G_{\mathrm{bp}}=\frac{2 \zeta \omega_{\beta} s}{s^{2}+2 \zeta \omega_{\beta} s+\omega_{\beta}^{2}}
$$

and complementary notch filter

$$
G_{\text {ntch }}=\frac{s^{2}+\omega_{\beta}^{2}}{s^{2}+2 \zeta \omega_{\beta} s+\omega_{\beta}^{2}}
$$

where $\omega_{\beta}$ is the disturbance frequency and $\zeta$ controls the bandwidth. $\zeta=0.01$ is appropriate for first- and third-order mains harmonics.

With the inclusion of periodic interference rejection, the filters $F_{c}, F_{p}$, and $F_{m}$ become

$$
\begin{aligned}
F_{c} & =G_{c}^{-1} \frac{\omega_{\alpha}}{s+\omega_{\alpha}} G_{\text {ntch }}^{1} \ldots G_{n t c h}^{n} \\
F_{p} & =\frac{s+\frac{1}{r_{p} c_{p}}}{s} \frac{s}{s+\omega_{\alpha}} G_{\text {ntch }}^{1} \ldots G_{\text {ntch }}^{n} \\
F_{m} & =G_{\mathrm{bp}}^{1}+\cdots+G_{b p}^{n}
\end{aligned}
$$




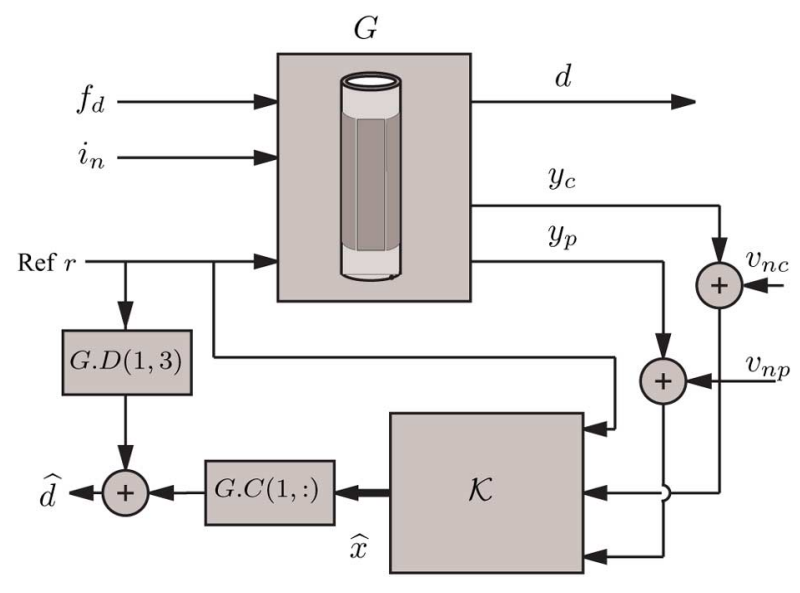

Fig. 8. Kalman displacement estimator $\mathcal{K}$. The displacement output matrix $C(1,:)$ and feedthrough $D(1,3)$ yield the estimated displacement $\widehat{d}$ from the optimal state estimation $\widehat{x} . f_{d}$ and $i_{n}$ are the disturbance force and measurement noise current, while $d, y_{c}$, and $y_{p}$ are the actual deflection, the capacitive sensor measurement, and the strain voltage measurement.

where $G_{\mathrm{bp}}^{n}$ and $G_{\text {ntch }}^{n}$ is the $n$th bandpass and notch filter, respectively. Two filters targeting the first and third mains frequency harmonics can remove up to $95 \%$ of in-bandwidth interference, and the only added cost is increased DSP complexity.

\section{Kalman Sensor Fusion}

A more automated choice of estimator is a linear observer or Kalman filter [25]. With the assumption of Gaussian distributed random disturbance and measurement noise, a Kalman filter provides the minimum variance state estimate. Fig. 8 illustrates the physical system (Fig. 6) "repackaged" as a system block diagram. The noise input $v_{n c}$ has been approximated as pure measurement noise, as this simplifies the design process with negligible error. The discretized system $G$ described below incorporates all of the mechanical and electrical dynamics including noise filters, sensor dynamics, and amplifier dynamics:

$$
x_{t+1}=\mathbf{A} x_{t}+\mathbf{B}\left[\begin{array}{c}
f \\
i_{n} \\
r
\end{array}\right], \quad\left[\begin{array}{c}
d \\
y_{c} \\
y_{p}
\end{array}\right]=\mathbf{C} x_{t}+\mathbf{D}\left[\begin{array}{c}
f \\
i_{n} \\
r
\end{array}\right]
$$

where $A, B, C$, and $D$ are the system matrices of $G$ procured, for example, by system identification or manipulation of the individual transfer functions. The MATLAB function connect (from the Control Systems Toolbox) is useful for this purpose. It should be noted that the output $d$ is not measured during operating conditions. $d$ is included in the model so that it can be used as a performance objective and is reconstructed from the estimated state.

Based on the covariance of the disturbance $\mathbf{Q}$ and measurement noise, $\mathbf{R}$ is defined as

$$
\mathbf{Q}=\operatorname{Cov}\left\{\left[\begin{array}{c}
f \\
i_{n}
\end{array}\right]\right\}, \quad \mathbf{R}=\operatorname{Cov}\left\{\left[\begin{array}{c}
v_{n c} \\
v_{n p}
\end{array}\right]\right\}
$$

where Cov is the covariance. A Kalman observer that minimizes

$$
J=\lim _{t \rightarrow \infty} E\left\{\left[x_{t}-\widehat{x_{t}}\right]\left[x_{t}-\widehat{x_{t}}\right]^{\prime}\right\}
$$

can be found through the solution of an algebraic Ricatti equation [26]. The magnitude of $\mathbf{Q}$ and $\mathbf{R}$ expresses the relative confidence in the measured variables and defines the frequency regions where each signal is dominant. Note that, as the noise $i_{n}$ is integrated, the Kalman filter will always have zero sensitivity to constant error in the measured piezoelectric strain voltage, which is a desirable property.

\section{E. Estimator Variance Improvement}

In many applications, the frequency-dependent nature of the capacitive and strain signals can be exploited to significantly improve the displacement estimate. One particular method can be used in cases where the desired scan trajectory consists of a large dc component and smaller high-frequency components, for example, a scanning pattern with offset. In this case, a lowfrequency large-range sensor is required but with no restriction on wideband noise as the control bandwidth associated with this signal can be extremely low. An additional low-range low-noise sensor should be used to provide wide bandwidth feedback for the dynamic part of the scan.

The capacitive sensor is ideal as a low-frequency full-range sensor and the piezoelectric strain signal is ideal as an ac coupled wideband low-range sensor. The range of the piezoelectric signal can be modified simply by switching in a different buffer input capacitance (as discussed in Section III-B). A buffer input capacitance of zero results in a signal with 62 times greater sensitivity than the capacitive sensor and almost negligible noise. The only required modification is an input capacitance change on the buffer circuit and a corresponding gain change in the control system. This technique allows a dynamic range far exceeding the usual $96 \mathrm{~dB}$ for $16 \mathrm{~b}$ with significantly less noise and bandwidth.

Further benefits are realized by manipulating the ac coupling frequency of the strain signal. The error induced by electrostatic and mechanical cross coupling from the adjacent axes can be eliminated. Because the fast scan axis of a scanning probe microscope operates hundreds to thousands of times faster than the slow scan axis, we can choose the ac coupling cutoff so that none of the slow scan signal appears at the strain voltage measurement.

\section{RECEDING HORIZON CONTROL}

The purpose of fusing the signals $y_{p}$ and $y_{c}$ is to obtain an estimate of the tube displacement $\hat{d}$ over a wide bandwidth for the purpose of improved tracking control. Here, we design a receding horizon controller that uses this state estimate in conjunction with a reference signal to provide a tracking control strategy. The major benefits of receding horizon control are: 1) it is inherently discrete and straightforward to implement in real-time; 2) it only places a penalty on tracking error over a finite horizon time not infinity as in LQG; and 3) it allows simultaneous design of feedforward and feedback controllers.

Alternative controllers include almost any state- or outputfeedback controller (based on $\widehat{d}$ ). One simple controller that was successfully tested but not reported here is a positive position feedback controller [27]. 


\section{A. Controller Design}

A receding horizon strategy results in the control law

$$
u_{t}=K \bar{x}_{t}
$$

where $\bar{x}_{t}$ is comprised of the Kalman state estimate in Section III-D and a vector containing samples of the reference input.

The receding horizon framework minimizes a cost function $V$ in order to obtain the next control action $u_{t+1}$. Then, at the next time interval, based on new measurements, the process is repeated again but this time generating $u_{t+2}$. For the purposes of this application, we consider the following cost:

$$
\begin{aligned}
V\left(u_{t+1 \mid t}, \ldots, u_{t+N \mid t}\right) \triangleq & \frac{1}{2} \sum_{k=1}^{N}\left\|\widehat{y}_{t+k \mid t}-r_{t+k}\right\|_{Q_{c}}^{2} \\
& +\left\|u_{t+k \mid t}-u_{t+k-1 \mid t}\right\|_{S}^{2} .
\end{aligned}
$$

In the above, $\widehat{y}_{t+k \mid t}$ is a prediction of the system output at some future time $t+k$ based on measurements up to and including the current time $t$. The quadratic form $\left\|\widehat{y}_{t+k \mid t}-r_{t+k}\right\|_{Q_{c}}^{2} \triangleq$ $\left(\widehat{y}_{t+k \mid t}-r_{t+k}\right)^{T} Q_{c}\left(\widehat{y}_{t+k \mid t}-r_{t+k}\right)$ is included to penalize deviations of the predicted output from future reference values $r_{t+k}$. Furthermore, the notation $u_{t+k \mid t}$ is used to highlight that these are future inputs based on information up to and including time $t$, and $\left\|u_{t+k \mid t}-u_{t+k-1 \mid t}\right\|_{S}^{2}$ is included to penalize control movements.

Therefore, at time $t$, we can compute the optimal sequence of control movements $\left\{u_{t+1 \mid t}^{\star}, \ldots, u_{t+N \mid t}^{\star}\right\}$ via

$\left\{u_{t+1 \mid t}^{\star}, \ldots, u_{t+N \mid t}^{\star}\right\}=\underset{u_{t+1 \mid t}, \ldots, u_{t+N \mid t}}{\operatorname{argmin}} V\left(u_{t+1 \mid t}, \ldots, u_{t+N \mid t}\right)$

and then apply $u_{t+1 \mid t}^{\star}$ at the next time interval $t+1$.

In order to compute $u_{t+1 \mid t}^{\star}$, we need to be able to predict the output $y_{t+k}$ for $k=1, \ldots, N$. To achieve this, we use the state-space model and Kalman filter from Section III-D. This immediately provides $\widehat{y}_{t+1 \mid t}$; however, we require estimates of the output over the entire prediction horizon from time $t+1$ until time $t+N$, which can be straightforwardly obtained via

$$
\begin{aligned}
\widehat{y}_{t+1 \mid t}= & C \widehat{x}_{t+1 \mid t}+D u_{t+1 \mid t} \\
\widehat{y}_{t+2 \mid t}= & C A \widehat{x}_{t+1 \mid t}+C B u_{t+1 \mid t}+D u_{t+2 \mid t} \\
& \vdots \\
\widehat{y}_{t+j \mid t}= & C A^{j-1} \widehat{x}_{t+1 \mid t}+C\left(\sum_{k=1}^{j-1} A^{j-k-1} B u_{t+k \mid t}\right) \\
& +D u_{t+j \mid t} .
\end{aligned}
$$

The key point to note is that each output prediction is a function of the initial state $\widehat{x}_{t+1 \mid t}$ and future inputs $u_{t+i \mid t}$ only. This series of output prediction equations can be stated in an equivalent but more convenient manner using matrix vector notation. Let

$$
\begin{aligned}
& R_{t} \triangleq\left[\begin{array}{c}
r_{t+1 \mid t} \\
\vdots \\
r_{t+N \mid t}
\end{array}\right] \\
& Y_{t} \triangleq\left[\begin{array}{c}
\widehat{y}_{t+1 \mid t} \\
\vdots \\
\widehat{y}_{t+N \mid t}
\end{array}\right] \\
& U_{t} \triangleq\left[\begin{array}{c}
u_{t+1 \mid t} \\
\vdots \\
u_{t+N \mid t}
\end{array}\right] \\
& \Lambda=\left[\begin{array}{c}
C \\
C A \\
C A^{2} \\
\vdots \\
C A^{N-1}
\end{array}\right] \\
& \Phi=\left[\begin{array}{ccccc}
D & & & & \\
C B & D & & & \\
C A B & C B & D & & \\
\vdots & & & \ddots & \\
C A^{N-2} B & \ldots & \cdots & C B & D
\end{array}\right] \text {. }
\end{aligned}
$$

Then

$$
Y_{t}=\Lambda \widehat{x}_{t+1 \mid t}+\Phi U_{t}
$$

This allows a more succinct expression for the cost function $V$ as

$$
V\left(U_{t}\right)=\frac{1}{2} U_{t}^{T} H U_{t}+U_{t}^{T} f
$$

The terms $H$ and $f$ are given by

$$
H \triangleq \Phi^{T} \bar{Q} \Phi+\bar{S} \quad f \triangleq \Gamma\left[\begin{array}{c}
\widehat{x}_{t+1 \mid t} \\
u_{t} \\
R_{t}
\end{array}\right]
$$

where

$$
\begin{aligned}
& \Gamma \triangleq\left[\begin{array}{lll}
\Phi^{T} \bar{Q} \Lambda & \Sigma & -\Phi^{T} \bar{Q}
\end{array}\right] \\
& \Sigma \triangleq\left[\begin{array}{ccc} 
& S & \\
\varnothing_{(N-1) n_{x} \times n_{u}}
\end{array}\right] \\
& \bar{Q} \triangleq\left[\begin{array}{cccc}
Q_{c} & & & \\
& Q_{c} & & \\
& & \ddots & \\
& & & Q_{c}
\end{array}\right] \\
& \bar{S} \triangleq\left[\begin{array}{ccccc}
2 S & -S & & \\
-S & 2 S & -S & & \\
& \ddots & \ddots & \ddots & \\
& & -S & 2 S & -S \\
& & & -S & S
\end{array}\right] .
\end{aligned}
$$


Finally, the optimal input sequence $U_{t}^{\star}$ can be obtained via

$$
\begin{aligned}
U_{t}^{\star} & =\arg \min _{U_{t}} \frac{1}{2} U_{t}^{T} H U_{t}+U_{t}^{T} f, \\
& =-H^{-1} f \\
& =\underbrace{-H^{-1} \Gamma}_{M}\left[\begin{array}{c}
\widehat{x}_{t+1 \mid t} \\
u_{t} \\
R_{t}
\end{array}\right] .
\end{aligned}
$$

Since $H$ and $\Gamma$ involve fixed matrices $A, B, C, D, Q_{c}$, and $S$, then the matrix $M$ can be computed once and stored. Furthermore, the receding horizon approach uses only the first input $u_{t+1 \mid t}^{\star}$ from $U_{t}^{\star}$, so it suffices to consider only the first $n_{u}$ rows of $M$. More precisely, the optimal control move $u_{t+1 \mid t}^{\star}$ is given by

$$
u_{t+1 \mid t}^{\star}=K\left[\begin{array}{c}
\widehat{x}_{t+1 \mid t} \\
u_{t} \\
R_{t}
\end{array}\right]
$$

where

$$
K \triangleq\left[\begin{array}{ll}
I_{n_{u}} & \varnothing_{n_{u} \times(N-1) n_{u}}
\end{array}\right] M .
$$

\section{B. Implementation of the Controller}

In order to procure a model of the system $G$ (see Fig. 8), the frequency response functions from $r$ to all outputs were acquired with an HP 35670A spectrum analyzer. The displacement $d$ was measured using a Polytec PI PSV300 laser vibrometer.

The dynamics of $G$ were obtained using a frequency-domain system identification algorithm [28]. ${ }^{2}$ The disturbance input $f_{d}$ was chosen equivalent to $r$, and the dynamics due to $i_{n}$ set as an integrator. The concatenated plant including disturbance inputs $f_{d}$ and $i_{n}$, the reference input $r$, the measured and reference outputs $y_{c}, y_{p}$ and $d$, and the performance output $z$, was assembled using the MATLAB function connect. As the control system is implemented digitally, a single delay is added to the reference input to account for conversion and processing delay.

The MATLAB function kalman (from the Control Systems Toolbox) was used to design the Kalman estimator $\mathcal{K}$. Due to the syntax of Kalman, a new system is required with outputs $y_{c}$ and $y_{p}$ and inputs reversed, i.e., $r$ is the first input, followed by $f_{d}$ and $i_{n}$. With $Q=\mathbf{I}$, and

$$
R=\alpha\left[\begin{array}{cc}
1 & 0 \\
0 & E\left\{i_{n}^{2}\right\}
\end{array}\right]
$$

$\alpha$ and $E\left\{i_{n}^{2}\right\}$ were determined by observing the Kalman filter transfer functions from inputs to estimated displacement. By plotting each frequency response, the regions where each signal is dominant can be clearly observed. As $E\left\{i_{n}^{2}\right\}$ is increased, the ac coupling frequency of $y_{p}$ is increased. In our experiments, this was set to approximately $10 \mathrm{~Hz}$ in order to maximize dynamic range and minimize the errors discussed in Sections III-B and $\mathrm{E}$.

In order to compute the controller gain matrix $K$ in (24) for the receding horizon controller detailed in Section IV, we need

\footnotetext{
${ }^{2} \mathrm{An}$ implementation of the multivariable frequency-domain subspace algorithm by McKelvey et al. [28] is available by contacting the first author.
}

to specify the parameters $N, Q_{c}, S$. The ratio of $Q_{c}$ to $S$ determines the compromise between tracking performance and controller bandwidth. As $Q_{c}$ is increased, the tracking performance improves at the expense of controller bandwidth. In this experiment, $N=200, Q_{c}=0.1$, and $S=0.2$, which results in a high-gain wideband controller. Improved noise performance could be achieved at the expense of bandwidth by reducing $Q_{c}$.

As the control signal (23) is a function of the future reference values $R_{t}$, the reference signal must either be known or delayed by the horizon length. In this work, $R_{t}$ is obtained by delaying $N$ samples of $r_{t}$.

\section{EXPERIMENTAL RESULTS}

Here, the estimator and controller of the previous section are experimentally applied to the piezoelectric tube apparatus shown in Fig. 1. The estimator and controller are implemented using the Real Time Workshop for MATLAB and a dSpace DS1103 DSP prototyping system.

In Sections V-A-V-F, a series of experiments are described that evaluate the noise and dynamic performance of the proposed control strategy. We begin in Section V-A by comparing the measurement noise of the capacitive sensor and estimated displacement. Open-loop tracking error is then quantified in Section V-B. The control loop is closed in Sections V-C and $\mathrm{V}-\mathrm{D}$, where noise and tracking error are evaluated, respectively. Performance robustness is investigated in Section V-E before a summary of results is given in Section V-F.

In the following experiments, tracking error is calculated by measuring the rms difference between the measured displacement and a straight line over $90 \%$ of the scan range. RMS noise is measured during scanning by fitting a fourth-order polynomial curve to $90 \%$ of the scan range and computing the rms difference between the curve and measured data. A fourth-order polynomial was found to sufficiently model actuator hysteresis present in the scan ranges under consideration.

\section{A. Open-Loop Sensor Noise}

The accuracy of the displacement estimator can be evaluated by performing a $1.6-\mu \mathrm{m}$ scan at 3 and $50 \mathrm{~Hz}$. The resulting capacitive sensor signal and estimated displacement are plotted in Fig. 9(a) and (c). An excellent agreement between the measured and estimated displacement can be observed. A smaller scan of $120 \mathrm{~nm}$, shown in Fig. 9(e), demonstrates the improved noise performance of the estimated displacement.

The open-loop noise of the capacitive sensor $y_{c}$ and estimated displacement $\widehat{d}$ are summarized in Table II. The capacitive sensor noise floor is $5 \mathrm{~nm} \mathrm{rms}$; when combined with the piezoelectric strain voltage, this drops to $0.34 \mathrm{~nm} \mathrm{rms}$. The lower noise value in the 120-nm scan is due to the absence of residual hysteresis, which is present in the $1.6-\mu \mathrm{m}$ scan. Noise measurements at $50 \mathrm{~Hz}$ were not possible due to the presence of vibration.

\section{B. Open-Loop Tracking Error}

The open-loop tracking errors are summarized in Table II. For $3-\mathrm{Hz}$ scan frequency, the error is clearly dominated by hysteresis at $1.6-\mu \mathrm{m}$ range and noise at $120 \mathrm{~nm}$. The tracking error for $50-\mathrm{Hz}$ scans is dominated by induced vibration. 
Open-loop

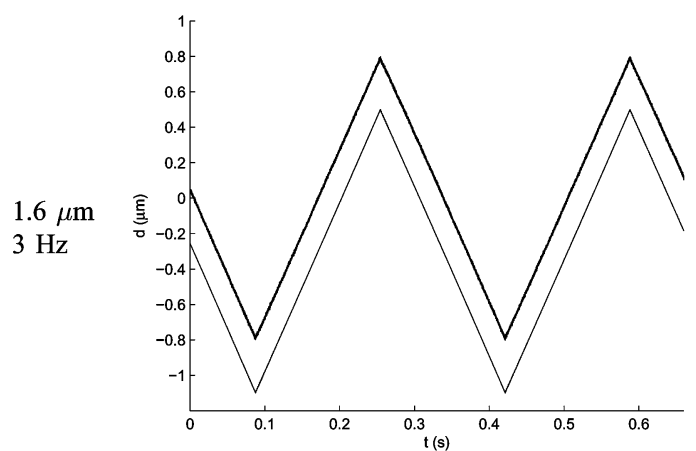

(a)

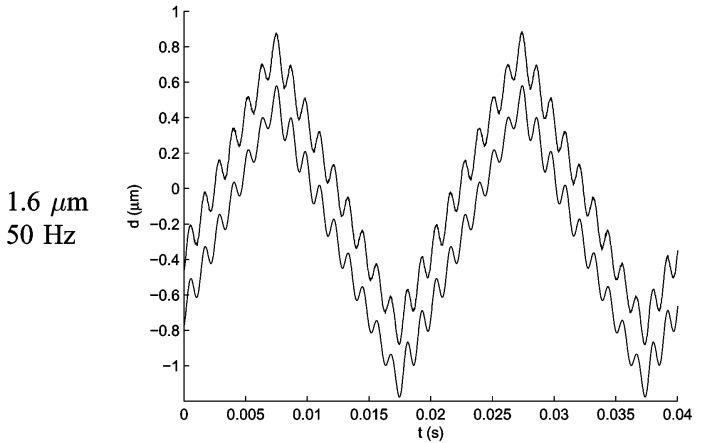

(c)

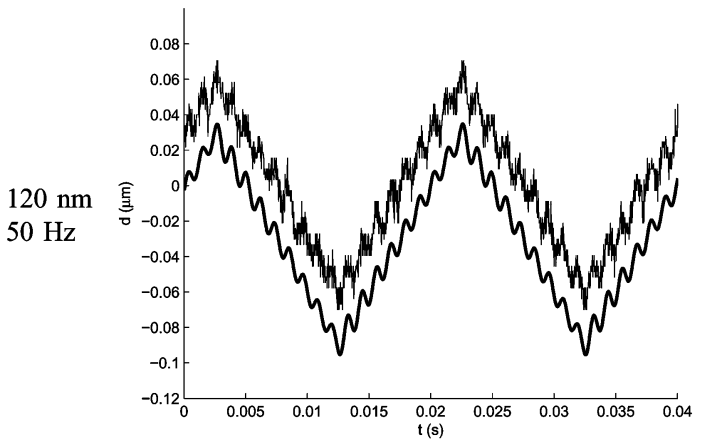

(e)
Closed Loop

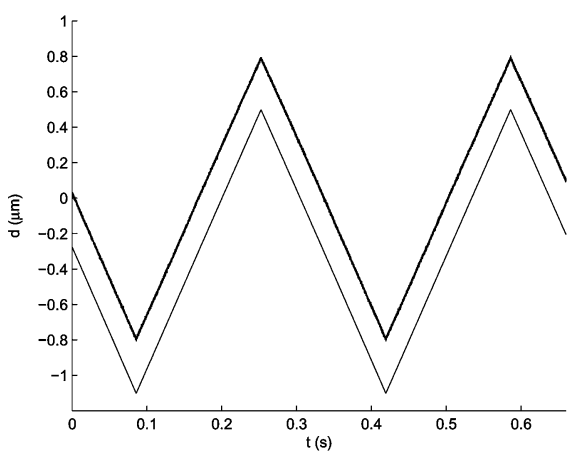

(b)

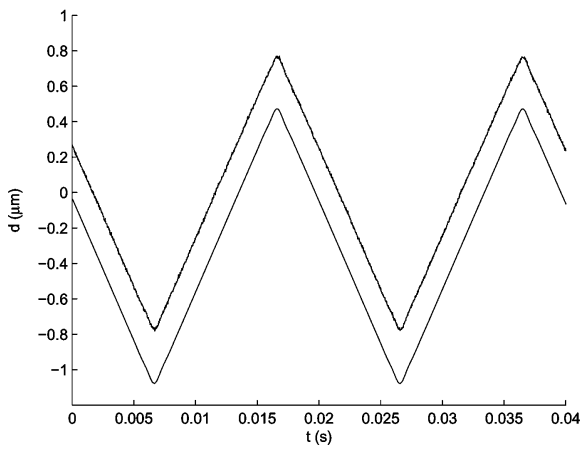

(d)

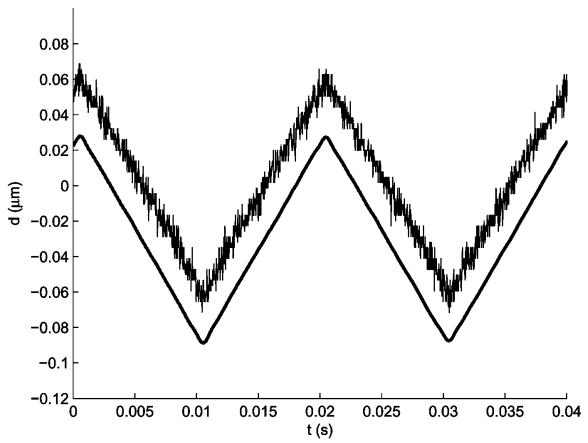

(f)

Fig. 9. Open- and closed-loop scan results at 3 and $50 \mathrm{~Hz}$ with ranges of $1.6 \mu \mathrm{m}$ and $120 \mathrm{~nm}$. Each plot contains the capacitive sensor signal (top) and the estimated displacement (bottom, with negative offset for clarity).

TABLE II

OPEN-LOOP NOISE AND TRACKING ERROR

\begin{tabular}{|l|l|l|l|}
\hline \multicolumn{5}{|c|}{ Open-loop noise } \\
\hline Scan Freq. & Scan Range & RMS $y_{c}$ & RMS $\widehat{d}$ \\
\hline $3 \mathrm{~Hz}$ & $1600 \mathrm{~nm}$ & $5.0 \mathrm{~nm}$ & $0.55 \mathrm{~nm}$ \\
\hline $3 \mathrm{~Hz}$ & $120 \mathrm{~nm}$ & $5.0 \mathrm{~nm}$ & $0.34 \mathrm{~nm}$ \\
\hline \hline \multicolumn{4}{|c|}{ Open-loop tracking error } \\
\hline Scan Freq. & Scan Range & RMS $y_{c}$ & RMS $\widehat{d}$ \\
\hline $3 \mathrm{~Hz}$ & $1600 \mathrm{~nm}$ & $5.0 \mathrm{~nm}$ & $1.0 \mathrm{~nm}$ \\
\hline $3 \mathrm{~Hz}$ & $120 \mathrm{~nm}$ & $5.0 \mathrm{~nm}$ & $0.35 \mathrm{~nm}$ \\
\hline $50 \mathrm{~Hz}$ & $1600 \mathrm{~nm}$ & $66.0 \mathrm{~nm}$ & $63.2 \mathrm{~nm}$ \\
\hline $50 \mathrm{~Hz}$ & $120 \mathrm{~nm}$ & $6.9 \mathrm{~nm}$ & $4.48 \mathrm{~nm}$ \\
\hline
\end{tabular}

\section{Closed-Loop Sensor Noise}

Although it is impossible to measure the scanner's closedloop noise directly (as we do not have a noise-free measurement), some insight can be gained by observing the sensor or estimated displacement noise in closed loop. In closed-loop, the noise appears to reduce as it is filtered by the sensitivity function of the control loop. Two methods of calculating the actual displacement noise are: estimation from the open-loop sensor noise and estimation from the closed-loop noise density function. Although this is straightforward for analog systems, in digital systems, such analysis is valid only when signal amplitudes are sufficiently large to avoid quantization noise. In the case of small amplitudes, the actual closed-loop noise is highly dependent on the signal magnitudes with respect to converter resolution. Unless sensor noise is significantly larger than quantization noise or a dither signal has been added [29], the most informative performance metric is the measured rms noise during practical modes of operation. Assuming that the bandwidth of the closed-loop system is smaller than the bandwidth of the estimated displacement, the true displacement noise can be upper 
TABLE III

CLOSED-LOOP NOISE AND TRACKING ERROR

\begin{tabular}{|l|l|l|l|}
\hline \multicolumn{5}{|c|}{ Closed-loop noise } \\
\hline Scan Freq. & Scan Range & RMS $y_{c}$ & RMS $\widehat{d}$ \\
\hline $3 \mathrm{~Hz}$ & $1600 \mathrm{~nm}$ & $4.9 \mathrm{~nm}$ & $0.36 \mathrm{~nm}$ \\
\hline $3 \mathrm{~Hz}$ & $120 \mathrm{~nm}$ & $5.1 \mathrm{~nm}$ & $0.36 \mathrm{~nm}$ \\
\hline $50 \mathrm{~Hz}$ & $1600 \mathrm{~nm}$ & $5.0 \mathrm{~nm}$ & $0.32 \mathrm{~nm}$ \\
\hline $50 \mathrm{~Hz}$ & $120 \mathrm{~nm}$ & $5.1 \mathrm{~nm}$ & $0.13 \mathrm{~nm}$ \\
\hline \hline \multicolumn{5}{|c|}{ Closed-loop tracking error } \\
\hline Scan Freq. & Scan Range & RMS $y_{c}$ & RMS $\widehat{d}$ \\
\hline $3 \mathrm{~Hz}$ & $1600 \mathrm{~nm}$ & $5.1 \mathrm{~nm}$ & $0.42 \mathrm{~nm}$ \\
\hline $3 \mathrm{~Hz}$ & $120 \mathrm{~nm}$ & $5.1 \mathrm{~nm}$ & $0.36 \mathrm{~nm}$ \\
\hline $50 \mathrm{~Hz}$ & $1600 \mathrm{~nm}$ & $5.2 \mathrm{~nm}$ & $0.41 \mathrm{~nm}$ \\
\hline $50 \mathrm{~Hz}$ & $120 \mathrm{~nm}$ & $5.2 \mathrm{~nm}$ & $0.29 \mathrm{~nm}$ \\
\hline
\end{tabular}

bounded by the measured closed-loop noise. This approach is taken here.

Table III lists the rms values of measured displacement noise. The loop is closed around $\widehat{d}$ only. In all of the experiments, the capacitive sensor noise remains at its nominal floor. In closedloop, the displacement noise reduces slightly compared with the open-loop. This reduction is due to the controllers action to track sensor noise within the control-loop bandwidth. The small magnitude of reduction follows from the low bandwidth of the sensitivity function $(800 \mathrm{~Hz})$ compared with the sensor bandwidth $(20 \mathrm{kHz})$. An approximate estimate for actual displacement noise is $0.07 \mathrm{~nm}$ rms (assuming an effective closed-loop bandwidth of $800 \mathrm{~Hz}$ and constant noise density). However, this value is only meaningful for scans that are sufficiently large to void quantization noise.

\section{Closed-Loop Tracking Error}

In Fig. 9(b), (d), and (f), the closed-loop response to 1.6- $\mu \mathrm{m}$ and $120-\mathrm{nm}$ scans are plotted. The controller provides satisfactory regulation even at high speed $(50 \mathrm{~Hz})$ and low amplitude $(120 \mathrm{~nm})$. Tracking error is summarized in Table III. Residual hysteresis causes the majority of tracking error in high-range scans $(1.6 \mu \mathrm{m})$, while residual vibration is the main source of error in high-frequency scans $(50 \mathrm{~Hz})$.

The closed-loop frequency response, which is plotted in Fig. 10, shows a 24-dB reduction of the resonance peak. When a $1.5 \mathrm{-g}$ mass is added to the tip, there is no degradation in damping performance.

\section{E. Performance Robustness}

From the frequency response plotted in Fig. 10, it is clear that the additional 1.5 -g mass has little effect on closed-loop performance. This is confirmed with a $1.6-\mu \mathrm{m}, 50-\mathrm{Hz}$ scan with additional mass shown in Fig. 11. The tracking performance is similar to that shown in Fig. 9 with the exception of a slightly degraded response at the signal apex. The degradation is attributed to the reduced system bandwidth when additional mass is present.

\section{F. Summary of Results}

The displacement estimator discussed in Section III-D results in a reduction of measurement noise from $5-\mathrm{nm}$ rms (for the

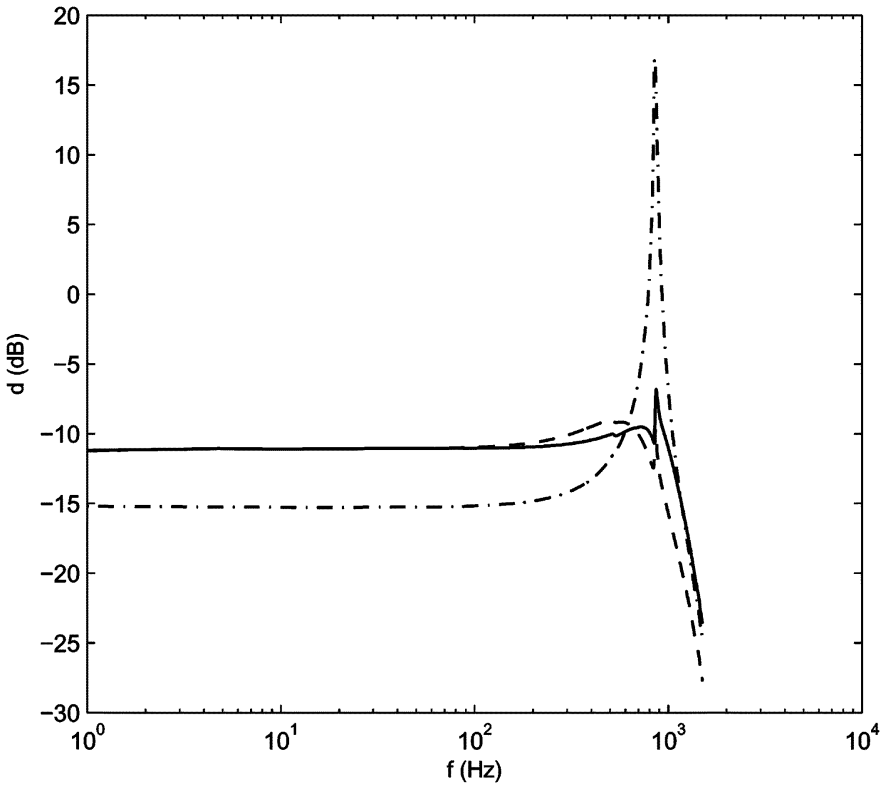

Fig. 10. Open- and closed-loop scanner frequency response measured from the reference input to the tip displacement (in micrometers per volts); open-loop $(-.-)$, unloaded (_ $(-)$, and with $1.5 \mathrm{~g}$ mass affixed $(--)$.

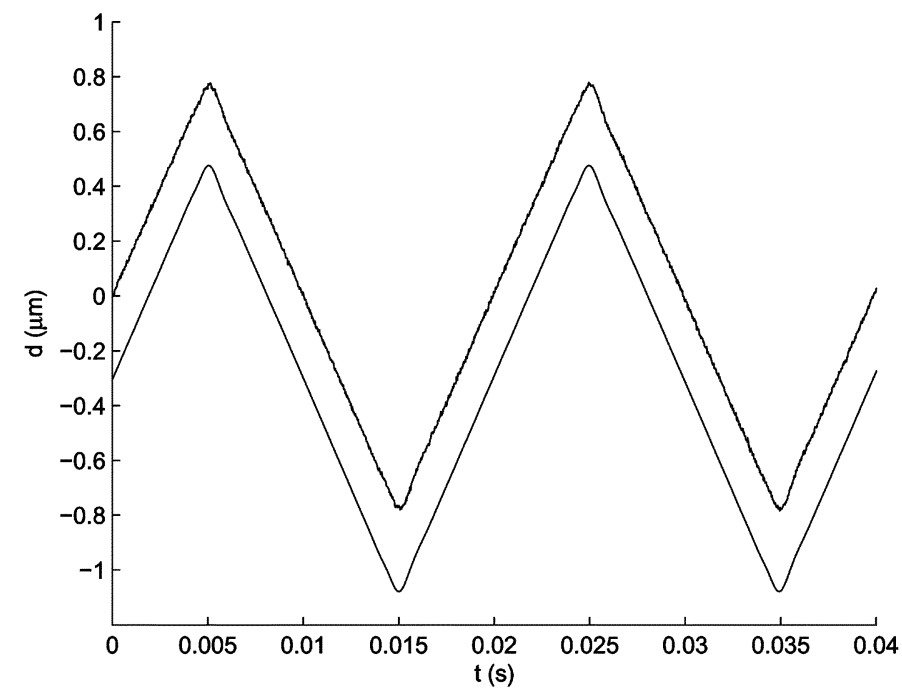

Fig. 11. 50-Hz 1.6- $\mu$ m closed-loop scan with additional $1.5 \mathrm{~g}$ mass; capacitive sensor (top), estimator (offset by $-0.3 \mu \mathrm{m}$ ).

capacitive sensor) to 0.34-nm rms, even though measurement bandwidth is extended to $20 \mathrm{kHz}$. Such low noise permits wide bandwidth control with subnanometer rms displacement noise.

A receding horizon controller was designed with a bandwidth sufficient to provide 24-dB damping of the first scanner resonance. At $50-\mathrm{Hz}$ scan frequency and $1.6-\mu \mathrm{m}$ range, the controller provides an excellent improvement in tracking error, from $65-\mathrm{nm}$ rms in open-loop to $0.41-\mathrm{nm}$ rms in closed-loop. Overall, the estimation and control strategy proved to be an excellent technique for obtaining higher tracking performance and lower noise than positioning systems utilizing capacitive sensors only. 


\section{CONCLUSION}

In addition to capacitive or inductive sensors, a piezoelectric strain sensor can provide large increases in measurement performance at little cost. One electrode per axis of a piezoelectric tube scanner can be utilized as a strain sensor while the other is retained for actuation. The only significant cost is the reduction in displacement range that would normally be achieved with a second voltage amplifier.

Although high-sensitivity piezoelectric materials, such as PZT-5H, exhibit significant temperature dependence and poor signal qualities at low frequencies, a technique is presented here to utilize only the desirable characteristics collaboratively with a capacitive sensor. With a model of the sensor dynamics and a linear estimator or Kalman filter, significant improvements to noise performance and dynamic range can be realized.

Experiments on a piezoelectric tube scanner, as found in scanning probe microscopes, demonstrated an rms displacement noise of $0.4 \mathrm{~nm}$ (sampled at $40 \mathrm{kHz}$ ) with a full scale range of $\pm 50 \mu \mathrm{m}$. The estimation technique lends itself easily to the inclusion of high-performance state-dependent controllers. A receding horizon control strategy was implemented that successfully attenuated scanner resonance by $24 \mathrm{~dB}$ without increasing displacement noise (within the limits of measurement). The controller was insensitive to the dominant uncertainty-large variations in resonance frequency.

Present and future work includes extending the technique to other nanopositioning applications where high dynamic range, low noise, and wide-bandwidth displacement feedback is required. Examples include kinematic stages with multiple axis and systems with different transducer arrangements, e.g., electromagnetically driven stages with strain and velocity feedback.

\section{REFERENCES}

[1] G. Binnig and D. P. E. Smith, "Single-tube three-dimensional scanner for scanning tunneling microscopy," Rev. Sci. Instrum., vol. 57, pp. 1688-1689, Aug. 1986.

[2] , D. A. Bonnell, Ed., Scanning Probe Microscopy and Spectroscopy-Theory, Techniques and Applications, 2nd ed. New York: Wiley, 2001.

[3] E. Meyer, H. J. Hug, and R. Bennewitz, Scanning Probe Microscopy. The Lab on a Tip. Heidelberg, Germany: Springer-Verlag, 2004.

[4] , B. Bhushan, Ed., The Handbook of Nanotechnology. Berlin, Germany: Springer-Verlag, 2004.

[5] A. A. Tsenga, A. Notargiacomob, and T. P. Chen, "Nanofabrication by scanning probe microscope lithography: A review," J. Vac. Sci. Technol., vol. 23, pp. 877-894, May/Jun. 2005.

[6] B. D. Gates, Q. Xu, J. C. Love, D. B. Wolfe, and G. M. Whitesides, "Unconventional nanofabrication," Аппи. Rev. Mater. Res., vol. 34, pp. 339-372, 2004.

[7] F. J. Rubio-Sierra, W. M. Heckle, and R. W. Stark, "Nanomanipulation by atomic force microscopy," Adv. Eng. Mater., vol. 7, pp. 193-196, 2005.

[8] W. Vogl, B. K.-L. Ma, and M. Sitti, "Augmented reality user interface for an atomic force microscope-based nanorobotic system," IEEE Trans. Nanotechnol., vol. 5, no. 4, pp. 397-406, Jul. 2006.

[9] S. Devasia, E. Eleftheriou, and S. O. R. Moheimani, "A survey of control issues in nanopositioning," IEEE Trans. Control Syst. Technol., vol. 15, no. 5, pp. 802-823, Sep. 2007.
[10] D. Croft, S. Stilson, and S. Devasia, "Optimal tracking of piezo-based nanopositioners," Nanotechnol., vol. 10, pp. 201-208, 1999.

[11] D. Croft, D. McAllister, and S. Devasia, "High-speed scanning of piezo-probes for nano-fabrication," Trans. ASME, J. Manuf. Sci. Technol., vol. 120, pp. 617-622, Aug. 1998.

[12] K. K. Leang and S. Devasia, "Design of hysteresis-compensating iterative learning control for piezo-positioners: Application to atomic force microscopes," Mechatron., vol. 16, pp. 141-158, 2006.

[13] Y. Wu and Q. Zou, "Iterative control approach to compensate for both the hysteresis and the dynamics effects of piezo actuators," IEEE Trans. Control Syst. Technol., vol. 15, no. 5, pp. 936-944, Sep. 2007.

[14] G. Schitter, R. W. Stark, and A. Stemmer, "Fast contact-mode atomic force microscopy on biological specimens by model-based control," Ultramicroscopy, vol. 100, pp. 253-257, 2004.

[15] D. Croft, G. Shed, and S. Devasia, "Creep, hysteresis, and vibration compensation for piezoactuators: Atomic force microscopy application," Trans. ASME, J. Dyn. Syst., Mea., Control, vol. 123, pp. 35-43, Mar. 2001.

[16] K. J. G. Hinnen, R. Fraanje, and M. Verhaegen, "The application of initial state correction in iterative learning control and the experimental validation on a piezoelectric tube scanner," J. Syst. Control Eng., vol. 218, pp. 503-511, 2004.

[17] G. Schitter and A. Stemmer, "Model-based signal conditioning for high-speed atomic force and friction force microscopy," Microelectron. Eng., vol. 67-68, pp. 938-944, 2003.

[18] H. Perez, Q. Zou, and S. Devasia, "Design and control of optimal scan trajectories: Scanning tunneling microscope example," J. Dyn. Syst., Meas., Control, vol. 126, pp. 187-197, Mar. 2004.

[19] N. Tamer and M. Dahleh, "Feedback control of piezoelectric tube scanners," in Proc. Amer. Control Conf., Lake Buena Vista, FL, Dec. 1994 pp. $1826-1831$.

[20] S. Salapaka, A. Sebastian, J. P. Cleveland, and M. V. Salapaka, "High bandwidth nano-positioner: A robust control approach," Rev. Sci. Instrum., vol. 75, pp. 3232-3241, Sep. 2002.

[21] C. J. Chen, "Electromechanical deflections of piezoelectric tubes with quartered electrodes," Appl. Phys. Lett., vol. 60, pp. 132-134, Jan. 1992.

[22] A. J. Fleming and S. O. R. Moheimani, "Sensorless vibration suppression and scan compensation for piezoelectric tube nanopositioners," IEEE Tran. Control Syst. Technol., vol. 14, no. 1, pp. 33-44, Jan. 2006.

[23] A. J. Fleming and S. O. R. Moheimani, "A grounded load charge amplifier for reducing hysteresis in piezoelectric tube scanners," Rev. Sci. Instrum., vol. 76, pp. 073707-073707, Jul. 2005.

[24] P. Horowitz and W. Hill, The Art of Electronics. Cambridge, U.K. Cambridge Univ. Press, 1989.

[25] R. G. Brown and P. Hwang, Introduction to Random Signals and Applied Kalman Filtering. New York: Wiley, 1997.

[26] S. Skogestad and I. Postlethwaite, Multivariable Feedback Control. New York: Wiley, 1996.

[27] J. L. Fanson and T. K. Caughey, "Positive position feedback control for large space structures," AIAA J., vol. 28, pp. 717-724, 1990.

[28] T. McKelvey, H. Akcay, and L. Ljung, "Subspace based multivariable system identification from frequency response data," IEEE Trans. Autom. Control, vol. 41, no. 7, pp. 960-978, Jul. 1996.

[29] P. Carbone and D. Petri, "Performance of stochastic and detarministic dithered quantizers," IEEE Trans. Instrum. Meas., vol. 49, no. 3, pp. 337-340, Apr. 2000.

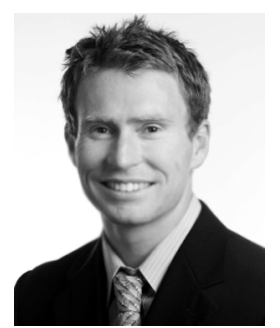

Andrew J. Fleming (M'03) was born in Dingwall, Scotland in 1977. He received the B.S. degree in electrical engineering and the Ph.D. degree from The University of Newcastle, Newcastle, Australia, in 2000 and 2004, respectively.

In 2005, he was a Research Academic with the Centre for Complex Dynamics and Control, The University of Newcastle. He is presently an Australian Research Council APD Fellow stationed at the School of Electrical Engineering and Computer Science, The University of Newcastle. His research includes micro-cantilever sensors, nano-positioning, and sensorless control of sound and vibration. 


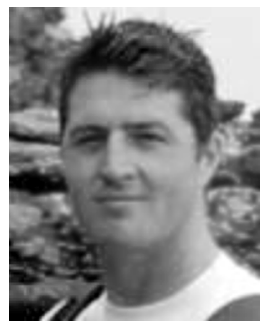

Adrian G. Wills was born in Orange, N.S.W. Australia. He received the B.E. and Ph.D. degrees from The University of Newcastle, Australia, in 1999 and 2003, respectively.

Since then, he has held a postdoctoral research position with The University of Newcastle, where the focus of his research has been in the area of system identification and model predictive control.

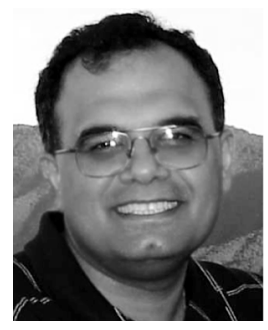

S. O. Reza Moheimani (SM'00) received the Ph.D. degree in electrical engineering from the University of New South Wales at the Australian Defence Force Academy, Canberra, Australia, in 1996.

He is currently a Professor with the School of Electrical Engineering and Computer Science, The University of Newcastle, Australia, where he serves as the Assistant Dean Research for the Faculty of Engineering and Built Environment. He has published two books, several edited volumes, and over 150 refereed articles in archival journals and conference proceed- ings. His current research interests include applications of control and estimation in nanoscale positioning systems for scanning probe microscopy, control of electrostatic microactuators in MEMS, and data storage systems. He is Associate Director of the ARC Centre for Complex Dynamic Systems and Control, an Australian Government Centre of Excellence. He has held several visiting appointments at IBM Zurich Research Laboratory, Switzerland.

Prof. Moheimani is a Fellow of the Institute of Physics, U.K. He was a recipient of the 2007 IEEE TRANSACtions ON CONTROL Systems TECHNOLOGY Outstanding Paper Award. He has served on the editorial board of a number of journals, including the IEEE TRANSACTIONS ON CONTROL SyStEMS TECHNOLOGY, and has chaired several international conferences and workshops. 